\title{
Imaging Small-animal Whole-body Dynamics by Single-impulse Panoramic Photoacoustic Computed Tomography
}

Lei Li ${ }^{\mathrm{a}}$, Liren Zhu ${ }^{\mathrm{a}}$, , Cheng $\mathrm{Ma}^{\mathrm{c}}$, Li Lin ${ }^{\mathrm{a}, \mathrm{b}}$, Junjie Yao ${ }^{\mathrm{d}}$, Lidai Wang ${ }^{\mathrm{e}}$, Konstantin Maslov ${ }^{\mathrm{a}}$, Ruiying Zhang ${ }^{\mathrm{b}}$, Wanyi Chen ${ }^{\mathrm{b}}$, Junhui Shi ${ }^{\mathrm{a}}$, Lihong V. Wang ${ }^{\mathrm{a}}$ *

${ }^{a}$ Department of Medical Engineering, California Institute of Technology, 1200 E California Blvd.

MC 136-93, Pasadena, CA 91125;

${ }^{b}$ Department of Biomedical Engineering, Washington University in St. Louis, One Brookings Dr.,

St. Louis, MO, 63130;

${ }^{\mathrm{c}}$ Department of Electronic Engineering, Tsinghua University, Beijing, 100084, China; ${ }^{\mathrm{d}}$ Department of Biomedical Engineering, Duke University, Durham, NC, 27708;

${ }^{d}$ Department of Biomedical Engineering, Duke University, Durham, NC, 27708;

${ }^{\mathrm{e}}$ Department of Mechanical and Biomedical Engineering, City University of Hong Kong, Kowloon, Hong Kong, China.

*Correspondence to: L.V.W. (LVW@Caltech.edu)

\begin{abstract}
Small animal whole-body imaging, providing physiological, pathological, and phenotypical insights into biological processes, is indispensable in preclinical research. With high spatiotemporal resolution and functional contrast, small animal imaging can visualize biological dynamics in vivo at whole-body scale, which can advance both fundamental biology and translational medicine. However, current non-optical imaging techniques lack either spatiotemporal resolution or functional contrasts, and pure optical imaging suffers from either shallow penetration (up to $\sim 1 \mathrm{~mm}$ ) or a poor resolution-to-depth ratio $(\sim 1 / 3)$. Here, we present a standalone system, termed single-impulse panoramic photoacoustic computed tomography (SIP-PACT), which overcomes all the above limitations. Our technology, with unprecedented performance, is envisioned to complement existing modalities for imaging entire small animals. As an optical imaging modality, SIP-PACT captures the high molecular contrast of endogenous substances such as hemoglobin, melanin, and lipid, as well as exogenous biomarkers, at the whole animal scale with full-view fidelity. Unlike other optical imaging methods, SIP-PACT sees through $\sim 5 \mathrm{~cm}$ of tissue in vivo, and acquires cross-sectional images with an in-plane resolution of $\sim 100 \mu \mathrm{m}$. Such capabilities allow us to image, for the first time, mouse wholebody dynamics in real time with clear sub-organ anatomical and functional details and without motion artifacts. SIPPACT can capture transients of whole-body oxygen saturation and pulse wave propagation in vivo without labeling. In sum, we expect widespread applications of SIP-PACT as a whole-body imaging tool for small animals in fundamental biology, pharmacology, pathology, oncology, and other areas.
\end{abstract}

Keywords: Single-impulse, whole-body imaging, small-animal imaging, photoacoustic computed tomography

\section{INTRODUCTION}

Small animals, especially rodents, are essential models for preclinical studies, and they play an important role in modeling human physiology and development, in guiding the study of human diseases, and in devising effective treatments [1]. Previously, small-animal whole-body imaging has typically relied on non-optical approaches, including magnetic resonance imaging (MRI), X-ray computed tomography (X-ray CT), positron emission tomography (PET) or single-photon emission computed tomography (SPECT), and ultrasound tomography (UST) $[2,3]$. Although these techniques provide deep penetration, they suffer from significant limitations. Optical imaging of biological tissue employs non-carcinogenic electromagnetic waves to provide extraordinary structural, functional, and molecular contrasts with either endogenous or exogenous agents [4-6]. Unfortunately, the application of conventional optical imaging technologies to small-animal whole-body imaging is impeded by the strong optical scattering of tissue, which prevents high-resolution imaging beyond the optical diffusion limit of $\sim 1-2 \mathrm{~mm}$ in depth [2]. To date, photoacoustic tomography (PAT) is the only high-resolution optical imaging modality that breaks the optical diffusion limit [7]. Photoacoustic

Photons Plus Ultrasound: Imaging and Sensing 2017, edited by Alexander A. Oraevsky, Lihong V. Wang, Proc. of SPIE Vol. 10064, 100640M - (C) 2017 SPIE · CCC code: 1605-7422/17/\$18 - doi: 10.1117/12.2251593 
computed tomography (PACT), a major incarnation of PAT, has provided penetration beyond $10 \mathrm{~mm}$, but with either poor temporal resolution, due to data acquisition multiplexing [8-10], or unclearly resolved sub-organ features, due either to partial acoustic detection coverage [11,12] or to sparse spatial sampling [13-15].

Here, we report a significant advance in PACT technology that overcomes all the above-mentioned limitations prevalent in both non-optical and pure optical imaging techniques. Our imaging technology, called single-impulse panoramic PACT (SIP-PACT), allows us to capture structural, functional, cellular, and molecular small-animal whole-body images with unprecedented speed and quality. SIP-PACT enables a number of new whole-body imaging capabilities. At a $50-\mathrm{Hz}$ frame rate, it non-invasively images whole-body small animals (up to $48 \mathrm{~mm}$ in width), with sub-organ vasculature and internal organ structures clearly resolved, without any labeling. At such high spatiotemporal resolutions, the biological dynamics associated with heartbeats and respiration are clearly observed without motion artifacts. Moreover, SIP-PACT detects the functional connectivity in the deep brain (up to $9.7 \mathrm{~mm}$ ).

\section{RESULTS}

\subsection{Label-free imaging of small-animal whole-body anatomy and dynamics}

In SIP-PACT, we employed a 512-element full-ring ultrasonic transducer array (Imasonic, Inc., 5-MHz central frequency, more than $90 \%$ one-way bandwidth) for 2D panoramic in-plane acoustic detection, which avoids artifacts induced by limited detection coverage [16]. The digitized raw data was fed into a half-time dual-speed-of-sound (in tissue and water) universal back-projection algorithm for image reconstruction. Top illumination and side detection were used for brain cortex imaging, and full-ring side illumination and side detection (aligned confocally to maximize detection sensitivity) were used for trunk imaging. To maximize optical penetration, we took advantage of the "optical window" at 650-1350 nm, where mammalian tissues least attenuate light. We used 1064-nm laser excitation with a 50$\mathrm{Hz}$ pulse repetition rate for this task. SIP-PACT non-invasively imaged the vasculature of the brain cortex (Fig. 1a) and the anatomy of the internal organs within the abdominal cavity (liver, spleen, kidney, cecum, and intestine; Fig. 1, b and c), with detailed structures revealed by hemoglobin contrast.

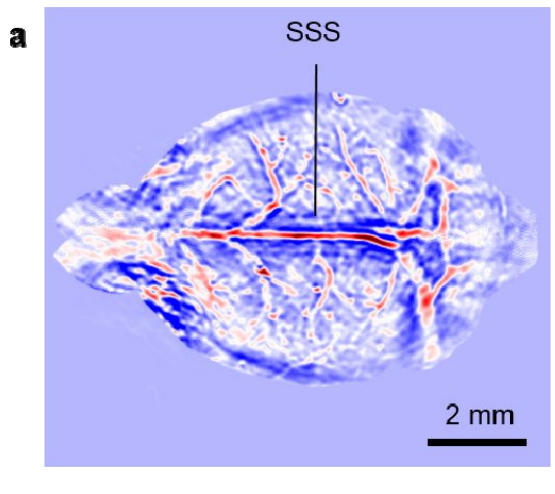

Min

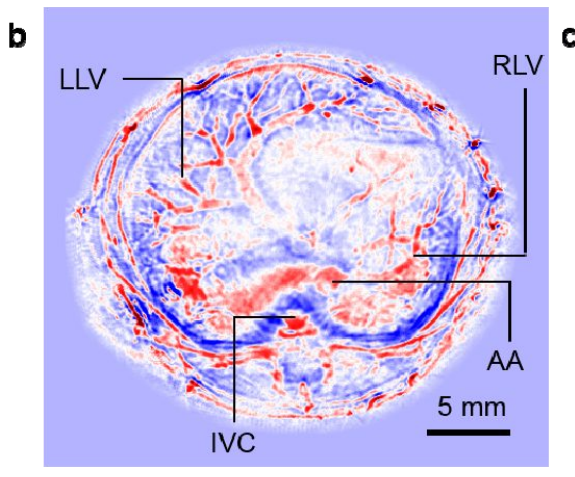

\section{PA amplitude}

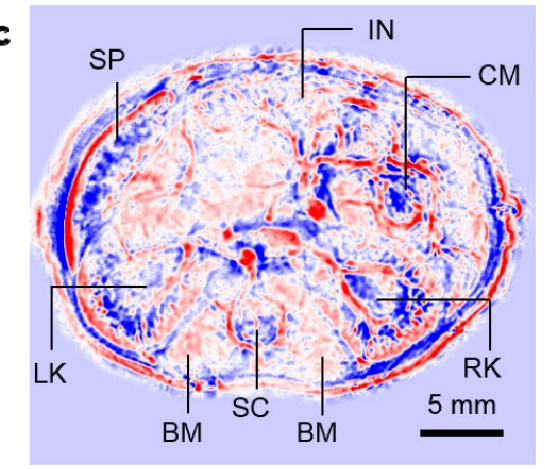

$\operatorname{Max}$

Figure 1. Label-free SIP-PACT of small-animal whole-body anatomy from the brain to the trunk. (a) Vasculature of the brain cortex; SSS, superior sagittal sinus. (b) Cross-sectional image of two lobes of liver; AA, abdominal aorta; IVC, inferior vena cava; LLV, left lobe of liver; PV, portal vein; RLV, right lobe of liver. (c) Cross-sectional imaging of lower abdominal cavity; BM, backbone muscles; CM, cecum; LK, left kidney; RK, right kidney.

At an imaging frame rate of $50 \mathrm{~Hz}$, respiratory motions and heartbeats were fully captured well above the Nyquist sampling rate. In one cross-sectional image of the thoracic cavity (Fig. 2a), both respiratory motion and heartbeats are displayed. By recording the signal changes of a rib (along the black solid line in Fig. 2a) and the heart wall (along the white dashed line in Fig. 3a), the respiratory motion and heartbeats could be tracked and identified (Fig. 2, b and c). Fourier analysis showed that the motion of the rib repeated at a respiratory frequency of $\sim 1 \mathrm{~Hz}$, and the motion of the heart wall repeated at both the respiratory frequency and a heartbeat frequency of $\sim 5.2 \mathrm{~Hz}$ (Fig. 3, d and e). These measurements were also validated by parallel monitoring results from electrocardiography (ECG) and a pressure sensor (Fig. 2, b to e). 
a

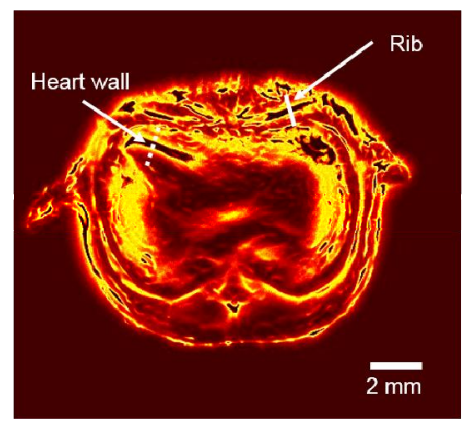

b

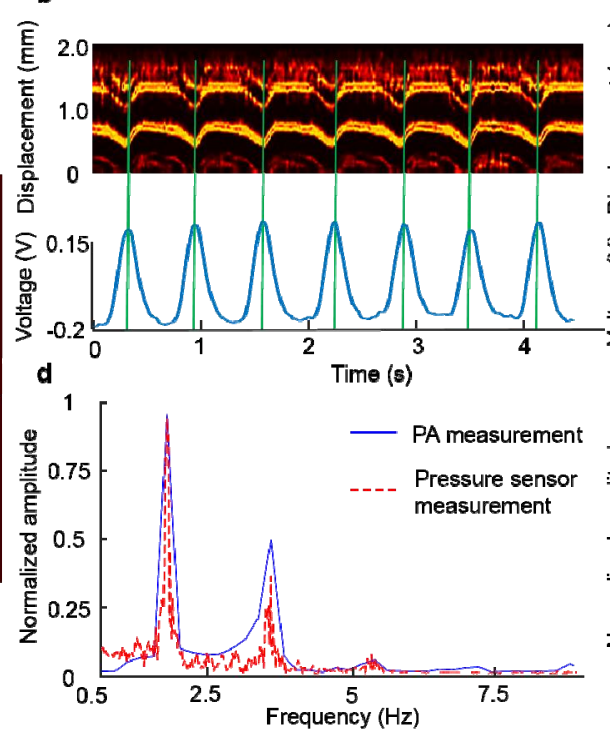

c

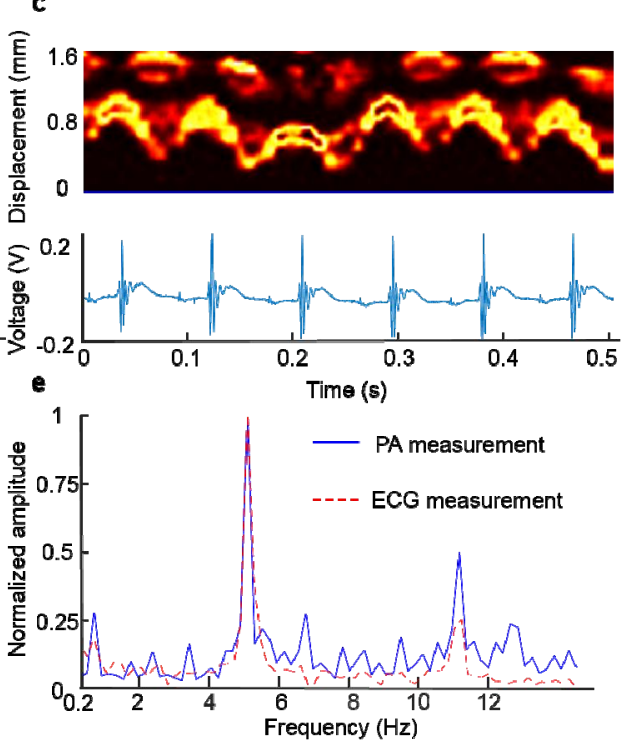

Figure 2. (a) Cross-sectional image of the upper thoracic cavity, where the black solid line crosses a rib, and the white dashed line crosses the heart wall. (b) Upper panel: line profiles in (a) versus time show the displacements of the rib during respiration; and lower panel: co-registered measurement of breathing motion from the pressure sensor. (c) Upper panel: line profiles in (a) versus time show the displacements of the heart wall during heartbeats. Lower panel: co-registered measurement of heartbeats from electrocardiography (ECG). (d) Fourier transform of (b), which shows identical respiratory frequency measurements by SIP-PACT and the pressure sensor. (e) Fourier transform of (c), which shows identical heartbeat frequency measurements by SIP-PACT and ECG.

Within a cross-sectional view of the lower abdominal cavity, the signals from arteries are temporally correlated due to their direct connection to the heart. The high imaging speed and the single-impulse acquisition capability of SIP-PACT enabled us to selectively map the arterial network on the whole-body cross-sectional image. In Fig. 3a, the arterial network is obtained by pixel-wise calculation of the amplitude at the heartbeat frequency, and the renal arteries of the right kidney are highlighted by heartbeat encoding. During systole, the aortic wall dilates due to the ejection of blood from the contracted left ventricle, generating a pressure wave that travels along the arterial tree. We selected two vertically distributed arteries from the arterial network (highlighted by arrows 1 and 2 in Fig. 3a) to compute the changes of the cross-sectional areas. A steady phase delay is revealed (Fig. 3b), indicating that the cross-sectional area changes are the results of the pulse wave propagating through the arterial network. This image demonstrates that SIP-PACT can noninvasively map the whole-body arterial network and measure the relative pulse wave phase difference between arteries. This capability could provide a non-invasive and direct diagnostic tool for chronic coronary artery disease and chronic renal disease $[17,18]$.

a

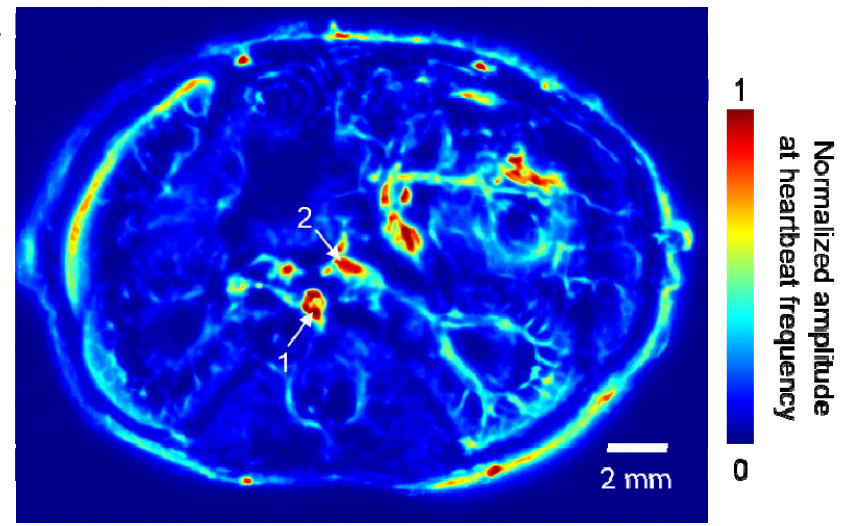

b

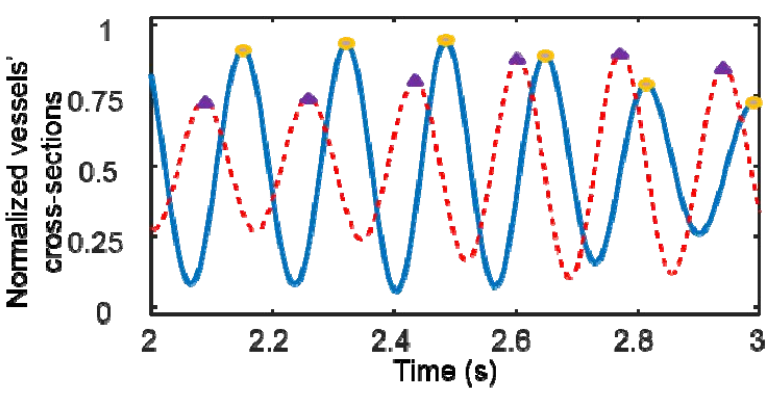

Figure 3. (a) Heartbeat encoded arterial network mapping overlaid on the anatomical image. (b) Cross-sections of the vessels highlighted by arrows in (a), showing changes associated with arterial pulse propagation. 


\subsection{Deep imaging through rat whole brain}

The brain, the center of the nervous system, dynamically coordinates responses through the functional network. The intrinsic functional connectivity (FC) across spatially separated brain regions can be measured through regionally correlated, spontaneous, low frequency $(0.01-0.1 \mathrm{~Hz})$ fluctuations in blood-oxygen-level-dependent (BOLD) signals with functional MRI (fMRI), particularly during resting-state/task-free periods (resting-state fMRI or rsfMRI). Similar to fMRI, SIP-PACT can also globally monitor the brain hemodynamics with appropriate spatiotemporal resolution and penetration. To detect the FC, we measured and compared the spontaneous hemodynamic responses between contralateral regions of the rat brain. We also employed a seed-based method to study the FC. The results (Fig. 4, a to c) show clear correlation between corresponding regions across the left and right hemispheres, as well as correlation between neighboring regions in the neocortex. These findings are consistent with previous research in both fMRI and fUS [19, 20]. Most interestingly, we imaged through the rat whole brain (11 $\mathrm{mm}$ in depth, Fig. 4d) and identified the left-right correlation between the deep thalamus regions $(9.7 \mathrm{~mm}$ in depth, Fig. $4, \mathrm{~d}$ to $\mathrm{f}$ ), which, to our best knowledge, has not been demonstrated at this spatial resolution. Our FC observation demonstrates the potential of SIP-PACT as a high-resolution imaging tool for studying deep brain functions in rats, which was previously difficult to accomplish using optical contrast, and, therefore underexplored.
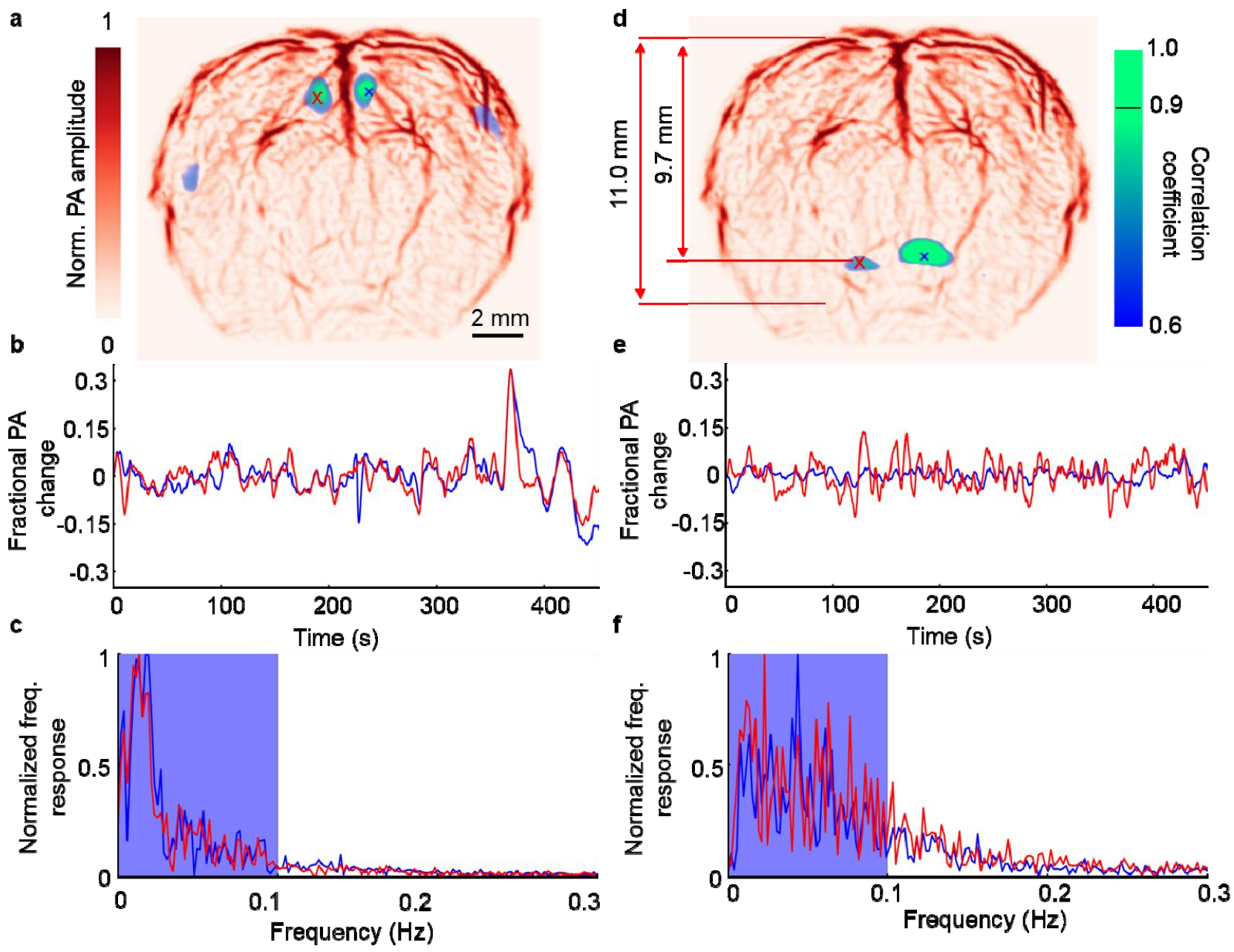

Figure 4. Spontaneous hemodynamic variations show high temporal correlation in contralateral regions in a rat brain. (a) Correlation map of the rat brain, with a right seed marked by a blue cross. (b) Spontaneous variations in the PA signal in the contralateral regions marked by the blue cross (blue curve) and red cross (red curve) in (a) show high temporal correlations. (c) Frequency distribution of the PA signals in the correlated regions in (a). (d-f) Similar to (a-c), another set of correlation regions in the deep brain $(9.7 \mathrm{~mm}$ in depth) and their temporal hemodynamic responses. 


\section{DISCUSSION}

Substantially enhanced imaging performance enables SIP-PACT to complement existing modalities for small-animal whole-body imaging. The SIP-PACT system offers a number of whole-body imaging capabilities. It non-invasively images the mouse anatomy in real time, with clearly resolved sub-organ vasculature and structures. With a frame rate of $50 \mathrm{~Hz}$, SIP-PACT reveals whole-body cardiac related dynamics and selectively maps the whole-body arterial network in mice. SIP-PACT has further extended the functional brain imaging depth to the rat whole brain $(\sim 10 \mathrm{~mm}$ in depth), which has not been demonstrated with optical contrast and spatiotemporal resolution as fine as SIP-PACT provides.

\section{ACKNOWLEDGMENTS}

We thank Yun He, Chiye Li, Yang Li, and Jun Xia for technical support, and James Ballard for close reading of the manuscript. This work was sponsored by the US National Institutes of Health grants DP1 EB016986 (NIH Director's Pioneer Award), R01 CA186567 (NIH Director's Transformative Research Award), U01 NS090579 (BRAIN Initiative), R01 EB016963, and S10 RR026922.

\section{REFERENCES}

[1] M. Baker, "Whole-animal imaging: The whole picture," Nature, 463(7283), 977-980 (2010).

[2] V. Ntziachristos, "Going deeper than microscopy: the optical imaging frontier in biology," Nature Methods, 7(8), 603-614 (2010).

[3] X. Jun, and L. V. Wang, "Small-Animal Whole-Body Photoacoustic Tomography: A Review," IEEE Transactions on Biomedical Engineering, 61(5), 1380-9 (2014).

[4] T. Kim, R. Zhou, M. Mir et al., "White-light diffraction tomography of unlabelled live cells," Nat Photon, 8(3), 256-263 (2014).

[5] N. G. Horton, K. Wang, D. Kobat et al., "In vivo three-photon microscopy of subcortical structures within an intact mouse brain," Nat Photon, 7(3), 205-209 (2013).

[6] F. Zhang, L.-P. Wang, M. Brauner et al., "Multimodal fast optical interrogation of neural circuitry," Nature, 446(7136), 633-639 (2007).

[7] D. Razansky, M. Distel, C. Vinegoni et al., "Multispectral opto-acoustic tomography of deep-seated fluorescent proteins in vivo," Nature Photonics, 3(7), 412-417 (2009).

[8] A. P. Jathoul, J. Laufer, O. Ogunlade et al., "Deep in vivo photoacoustic imaging of mammalian tissues using a tyrosinase-based genetic reporter," Nature Photonics, 9(4), 239-246 (2015).

[9] J. Yao, A. A. Kaberniuk, L. Li et al., "Multiscale photoacoustic tomography using reversibly switchable bacterial phytochrome as a near-infrared photochromic probe," Nature Methods, 13(1), 67-+ (2016).

[10] H.-P. Brecht, R. Su, M. Fronheiser et al., "Whole-body three-dimensional optoacoustic tomography system for small animals," Journal of Biomedical Optics, 14(6), 064007-064007-8 (2009).

[11] A. Taruttis, S. Morscher, N. C. Burton et al., "Fast Multispectral Optoacoustic Tomography (MSOT) for Dynamic Imaging of Pharmacokinetics and Biodistribution in Multiple Organs," PLOS ONE, 7(1), e30491 (2012).

[12] E. Merčep, N. C. Burton, J. Claussen et al., "Whole-body live mouse imaging by hybrid reflection-mode ultrasound and optoacoustic tomography," Optics Letters, 40(20), 4643-4646 (2015).

[13] D. Razansky, A. Buehler, and V. Ntziachristos, "Volumetric real-time multispectral optoacoustic tomography of biomarkers," Nat. Protocols, 6(8), 1121-1129 (2011).

[14] X. Luis Dean-Ben, and D. Razansky, "Adding fifth dimension to optoacoustic imaging: volumetric timeresolved spectrally enriched tomography," Light Sci Appl, 3, e137 (2014).

[15] J. Tang, J. E. Coleman, X. Dai et al., "Wearable 3-D Photoacoustic Tomography for Functional Brain Imaging in Behaving Rats," Scientific reports, 6, (2016). 
[16] X. Yuan, and L. V. Wang, "Effects of acoustic heterogeneity in breast thermoacoustic tomography," Ultrasonics, Ferroelectrics, and Frequency Control, IEEE Transactions on, 50(9), 1134-1146 (2003).

[17] J. N. Cohn, S. Finkelstein, G. McVeigh et al., "Noninvasive pulse-wave analysis for the early detection of vascular-disease," Hypertension, 26(3), 503-508 (1995).

[18] E. Kis, O. Cseprekal, Z. Horvath et al., "Pulse wave velocity in end-stage renal disease: Influence of age and body dimensions," Pediatric Research, 63(1), 95-98 (2008).

[19] E. Jonckers, J. Van Audekerke, G. De Visscher et al., "Functional Connectivity fMRI of the Rodent Brain: Comparison of Functional Connectivity Networks in Rat and Mouse," PLOS ONE, 6(4), e18876 (2011).

[20] B.-F. Osmanski, S. Pezet, A. Ricobaraza et al., "Functional ultrasound imaging of intrinsic connectivity in the living rat brain with high spatiotemporal resolution," Nature communications, 5, (2014). 